\section{Die Isotopen-Zusammensetzung von Strontium aus Meerwasser und aus rubidium-armen Gesteinen}

\author{
Von H. Ewald, S. Garbe und P. NEY * \\ Physikalisches Institut der Technischen Hochschule \\ München \\ (Z. Naturforschg. 11 a, 521-522 [1956] ; eingegangen am 3. Mai 1956)
}

Für zehn Strontium-Proben, die aus Meerwasser, Strontianiten, Cölestinen und Kalksteinen verschiedener geologischer Herkunft abgetrennt wurden, wurden massenspektrometrisch die Häufigkeitsverhältnisse der Isotope 86,87 und 88 bestimmt. Die in Tab. 1 enthaltenen Ergebnisse sind Mittelwerte aus jeweils etwa 20 bis 40 Einzelregistrierungen. Die aus den Schwankungen der Einzelmessungen errechneten wahrscheinlichen Fehler der Mittelwerte der Häufigkeitsverhältnisse ${ }^{86} \mathrm{Sr} /{ }^{88} \mathrm{Sr}$ und ${ }^{87} \mathrm{Sr} /{ }^{86} \mathrm{Sr}$ wurden mit Sicherheitsfaktoren 3 bzw. 4 multipliziert angegeben.

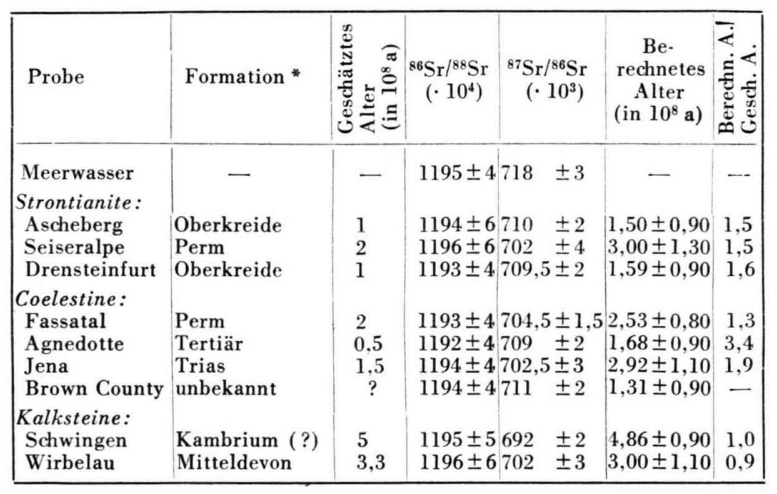

* Bei den Strontianiten und Coelestinen Formation der Muttergesteine

Tab. 1.

Die Ergebnisse für das Verhältnis ${ }^{86} \mathrm{Sr} /{ }^{88} \mathrm{Sr}$ stimmen bei diesen Proben im Rahmen der angegebenen wahrscheinlichen Fehler bis auf wenige Promille miteinander überein. Aldrich, Herzog u. a. ${ }^{1,2}$ berichteten dagegen 1953 über Unterschiede dieser Verhältniswerte für Proben verschiedener geologischer Herkunft, die die Größe von einigen Prozenten erreichten. Je zwei der von diesen Autoren und jetzt von uns untersuchten Proben sind gleicher Art und Herkunft (Cölestine aus Jena/Thüringen und aus Brown County/Texas). Herzog, Aldrich u. a. ${ }^{2}$ fanden bei diesen beiden Proben für das Verhältnis ${ }^{86} \mathrm{Sr} /{ }^{88} \mathrm{Sr}$ die Werte 0,1202 bzw.

* Letzterer bislang Mineralogisches Institut der Technischen Hochschule München, jetzt Materialprüfungsamt für das Bauwesen, TH München.

1 L. T. Aldrich, L. F. Herzog, J. B. Doak u. G. L. Davis, Trans. Amer. Geophys. Union 34, 457 [1953].

2 L. F. Herzog, L. T. Aldrich, W. K. Holyk, F. B. Whiting u. L. H. Ahrens, Trans. Amer. Geophys. Union 34, 461 [1953]. a L. T. Aldrich, L. F. Herzog, W. H. Pinson u. G. L. Davis, Trans. Amer. Geophys. Union 36, 875 [1955].
0,1221, sie stellen die Richtigkeit ihrer damaligen Ergebnisse in einer neuen Mitteilung ${ }^{2 a}$ aber in Frage.

Beim Verhältnis ${ }^{87} \mathrm{Sr} /{ }^{86} \mathrm{Sr}$ wurden, wie zu erwarten $^{3,4,5,6}$, Unterschiede von einigen Prozenten zwischen den Ergebnissen für die verschiedenen Proben gemessen (Tab. 1). Herzog u. a. ${ }^{2}$ fanden für dieses Verhältnis ebenfalls Schwankungen derselben Größenordnung. Für die Cölestine aus Jena und Brown County erhielten sie die Werte 0,709 bzw. 0,711.

Die Meerwasser-Probe weist den relativ höchsten Gehalt an ${ }^{87} \mathrm{Sr}$ auf. Dies ist im Vergleich zu StrontiumProben aus rubidium-armen Meeressedimenten verschiedenen geologischen Alters auch zu erwarten. Die Kalksteine sind meist solche Meeressedimente, während die Cölestine und Strontianite hydrothermaler oder lateralsekretionärer Herkunft sein können.

Aus den verschiedenen ${ }^{87} \mathrm{Sr} /{ }^{86} \mathrm{Sr}$-Verhältnissen haben wir nach der von $\mathrm{W}_{\text {IскмаN }}{ }^{3}$ und in ähnlicher Weise von $\mathrm{W}_{\text {ALLING }}{ }^{4}$ vorgeschlagenen Strontiumisotopen-Methode ${ }^{5,6}$ versuchsweise Alterszahlen für die Gesteine abgeleitet. Für das dabei eingehende geochemische ${ }^{87} \mathrm{Rb} /{ }^{87} \mathrm{Sr}-\mathrm{Ver}$ hältnis haben wir 4,62 gesetzt $^{6}$, für die Zerfallskonstante $^{7}$ des ${ }^{87} \mathrm{Rb} \lambda=1,61 \cdot 10^{-11} \mathrm{a}^{-1}$. Da für beide Größen in der Literatur erheblich unterschiedliche Werte angegeben wurden, können die erhaltenen Alterszahlen zunächst nur als Relativzahlen einen gewissen Wert beanspruchen. Aber auch unter Berücksichtigung dieser Tatsache ergibt ein Vergleich mit den Alterszahlen, wie sie für dieselben Gesteine auf Grund ihrer geologischen Formation geschätzt werden (Tab. 1), keine gute Übereinstimmung. Die Verhältnisse von errechneten zu geschätzten Alterszahlen streuen zwischen 0,9 und 3,4. Das ist bei der meist unsicheren genetischen Vorgeschichte der Strontianite und Cölestine nicht allzu verwunderlich. Bei den Kalksteinen mag noch am ehesten Übereinstimmung zu erwarten sein, weil es sich bei ihnen meist um im Stoffbestand unveränderte Sedimente handelt.

Die in Tab. 1 mit angegebenen wahrscheinlichen Fehler der errechneten Alterszahlen resultieren allein aus den Fehlern der gemessenen Häufigkeitsverhältnisse des Meerwasser-Strontiums und der Strontium-Proben aus den Gesteinen. Da wir die Zahlen zunächst nur als Relativzahlen werten wollten, haben wir für das geochemische ${ }^{87} \mathrm{Rb} /{ }^{87} \mathrm{Sr}$-Verhältnis und die Zerfallskonstante des ${ }^{87} \mathrm{Rb}$ obengenannte Werte als Festwerte ohne Fehler verwendet.

Eine ausführliche Veröffentlichung hierüber wird an anderer Stelle erscheinen. Die Untersuchungen werden vorzugsweise an Kalksteinen fortgesetzt.

Herrn Professor W. Borchert danken wir dafür, daß er die Arbeitsmöglichkeiten des Mineralogischen Insti-

3 F. E. Wickman, J. Geol. 56, 61 [1948].

4 E. W

5 H. Ewald u. H. Hintenberger, Methoden und Anwendungen der Massenspektroskopie, Verlag Chemie, Weinheim 1953, S. 231.

6 K. Rankama, Isotope Geology, Pergamon Press Ltd., London 1954, S. 336.

7 I. GeEse-B̈̈нNisch, Z. Phys. 142, 565 [1955]. 
tuts der Technischen Hochschule zur Verfügung stellte, Herrn Professor F. Rost vom gleichen Institut für hilfreiche Diskussionen. Dem B und d e r Fre und e der Technischen Hochschule München danken wir für die
Bereitstellung von Mitteln zum Aufbau des Massen. spektrometers. Auch der Deutschen Forschungs g emeinsch aft sind wir für finanzielle Unterstützung dieser Untersuchungen zu Dank verpflichtet.

\section{Über die Existenz der Zener-Emission}

\section{Von Walter Franz}

Institut für theoretische Physik der Universität Münster (Z. Naturforschg. 11 a, 522-523 [1956]; eingegangen am 18. Mai 1956)

WANNIER $^{1}$ gelangte kürzlich auf Grund einer Abänderung der Methode von Houston ${ }^{2}$ zu dem Schluß, daß innere Feldemission aus dem Valenzband (ZENER-Emission) theoretisch nicht existiert. Dieses Ergebnis erscheint physikalisch unglaubwürdig, da nicht einzusehen ist, weshalb eine Elektronenwelle nicht gedämpft die endliche Wegstrecke zurücklegen soll, welche benötigt wird, um aus dem äußeren elektrischen Feld die zu seiner Befreiung nötige Energie aufzunehmen. In der Tat zeigt eine nähere Untersuchung der $\mathrm{W}_{\text {ANNIERschen }} \mathrm{Ar}$ beit, daß der angebliche Beweis für die Nichtexistenz des Zener-Effekts sich nicht halten läßt.

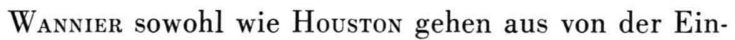
elektronen-SchröDINGER-Gleichung

$$
\left[-\frac{\hbar^{2}}{2 m} \Delta+V(\mathfrak{r})+e \mathfrak{F} \cdot \mathfrak{r}+\frac{\hbar}{i} \frac{\partial}{\partial t}\right] \chi(\mathfrak{r}, t)=0
$$

eines Elektrons im periodischen Gitterpotential $V(\mathrm{r})$ mit dem zusätzlichen Potential eines homogenen elektrischen Feldes $e \mathfrak{F} \cdot \mathfrak{r}$, welche sie durch Funktionen von folgendem Typus lösen:

$$
\chi(\mathrm{k}, \mathrm{r}, t)=u(\Re, \mathrm{r}) \exp \left[i \Re \cdot \mathrm{r}-\frac{i}{\hbar} \int^{t} E(\Re(\tau)) \mathrm{d} \tau\right] .
$$

$\mathfrak{K}$ ist dabei ein durch das Feld zeitlich veränderter Wellenzahlvektor

$$
\Re=k-\frac{e \mathfrak{F}}{\hbar} t
$$

und $u(\Re, \mathrm{r})$ besitzt die Periodizität der BLochschen Wellenfunktionen des Bandelektrons. Während Houston genau die B Bochsche Bandfunktion als Anfangszustand zur Zeit $t=0$ annimmt und dadurch unter dem Einfluß des elektrischen Feldes Übergänge in höhere Bänder erhält, weist $\mathrm{W}_{\text {ANNIER }}$ darauf hin, daß man mit Funktionen der Gestalt (2) Gl. (1) exakt lösen kann, wenn man $u$ der Wellengleichung

$$
\left[\frac{\hbar^{2}}{2 m}\left(\mathfrak{k}-i \frac{\partial}{\partial \mathfrak{r}}\right)^{2}+V(\mathrm{r})+i e \mathfrak{F} \cdot \frac{\partial}{\partial \mathfrak{k}}-E(\mathfrak{k})\right] u(\mathfrak{k}, \mathrm{r})=0
$$

unterwirft, welche sich von der BLochschen Wellengleichung durch den Feldterm unterscheidet; dabei ergeben sich keine Übergänge in höhere Bänder, die Zener-Emission würde also nicht existieren.

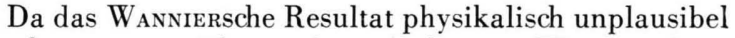
ist, hat man zu überprüfen, ob die von $\mathrm{W}_{\text {ANNIER }}$ bzw.
Houston benützten nichtstationären Wellenfunktionen überhaupt zur Beschreibung eines vollbesetztes Bandes im homogenen elektrischen Feld geeignet sind. $\mathrm{Zu}$ diesem Zweck hat man nach der „besten“ DeterminantenEigenfunktion des Vielelektronen-Problems zu suchen, deren Einelektronen-Funktionen von der Gestalt (2) sind. Da die Transformation (3) der k-Vektoren nur eine zyklische Permutation der Zustände des vollbesetzten Bandes bedeutet, welche die Determinantenfunktion nicht verändert, ist diese von der Gestalt

$$
\Psi=\left|u\left(\mathfrak{k}_{i}, \mathfrak{r}_{j}\right) \exp \left[i \mathfrak{k}_{i} \cdot \mathfrak{r}_{j}\right]\right| \exp \left[-\frac{i}{\hbar} E t\right] .
$$

Jede Zeile der Determinante gehört zu einem Zustand, welcher durch $k_{i}$ (sowie durch den nicht eigens angeschriebenen Spin) charakterisiert ist, während die Kolonnen je eine Elektronenkoordinate $\mathfrak{r}_{j}$ enthalten. Damit $k$ den durch (3) geforderten kontinuierlichen Wertevorrat besitzt, muß man zum Limes unendlich vieler Elektronen übergehen; die Determinante (5) erhält dabei unendlich viele Reihen und Kolonnen und wird hinsichtlich $\mathfrak{k}_{i}$ kontinuierlich. $E$ ist die zeitlich konstante Gesamtenergie des Bandes.

Die Bestimmung derjenigen Determinanten-Wellenfunktion, welche die Energie des Vielelektronen-Problems zu einem Minimum macht, führt bekanntlich auf die Fockschen Gleichungen. Da wir im Ansatz (5) jedoch durch die BLochsche Periodizitäts-Forderung die Mannigfaltigkeit der Einelektronen-Funktionen eingeschränkt haben, ergeben sich nicht ohne weiteres die Fockschen Gleichungen; durch das Potential des homogenen Feldes wird nämlich die Periodizität zerstört. Der Beitrag dieses Potentials zur Energie ist

$$
\int e \mathfrak{F} \cdot \mathrm{r} \sum_{i}\left|u\left(k_{i}, \mathrm{r}\right)\right|^{2} \mathrm{~d}^{3} \mathrm{r} .
$$

Als räumliches Volumen legen wir einen großen Periodizitäts-Kubus zugrunde, welcher aus einer ganzen Anzahl von Elementarzellen besteht. Die Schwerpunkte der Elementarzellen mögen bei den Gittervektoren $\mathfrak{g}$ liegen. Wir zerlegen nun in jeder einzelnen Elementarzelle das Potential in folgender Weise:

$$
e \mathfrak{F} \cdot \mathrm{r}=e \mathfrak{F} \cdot \mathrm{g}+e \mathfrak{F} \cdot(\mathrm{r}-\mathrm{g}) .
$$

Der erste Summand ist ein Stufenpotential, das in jeder Elementarzelle konstant ist; der zweite Summand ergibt ein im Gitter periodisches Sägezahn-Potential. Da die Anzahl der Elektronen einer Elementarzelle fest vorgegeben ist, wird der Beitrag des Stufenpotentials zu (6) von der Wahl der Funktionen $u$ unabhängig. Somit geht das Stufenpotential nicht in die Bestimmung der „besten“ Determinantenfunktion ein. Das SägezahnPotential andererseits hat die Periodizität des Gitters, 